\title{
Análisis del control prenatal que se brinda a las gestantes de la provincia de Heredia que tuvieron su parto en el Hospital San Vicente de Paúl ${ }^{1}$
}

Institución: Hospital San Vicente de Paúl

\author{
Nathalie Alfaro Vargas ${ }^{2}$ \\ Grettel Campos Vargas ${ }^{3}$
}

\section{COMO CITAR}

Alfaro, N. y Campos, G. (2014). Análisis del control prenatal que se brinda a las gestantes de la provincia de Heredia que tienen su parto en el Hospital San Vicente de Paúl, Heredia, durante el año 2012. Rev. Enfermería Actual en Costa Rica, 26, 1-19. Recuperado de: $<$ http://www.revenf.ucr.ac.cr/cuidado.pdf> ISSN 1409-4568

\section{RESUMEN}

Este artículo presenta los principales resultados de una investigación cuantitativa, con un diseño no experimental de tipo descriptivo transversal, la cual tuvo por objetivo analizar el control prenatal que se brinda a las gestantes de la provincia de Heredia que tuvieron su parto en el Hospital San Vicente de Paúl durante el año 2012. La población estuvo constituida por las gestantes que tuvieron su parto en dicho hospital entre los meses de diciembre 2011 a noviembre 2012 y por los profesionales en medicina y enfermería que laboran para las Áreas de Salud de Heredia y el Hospital San Vicente de Paúl, que brindan control prenatal. Para la recolección de la información se utilizaron tres instrumentos que consideraron la infraestructura, el equipamiento y el procedimiento para llevar a cabo el control prenatal, además, el nivel de satisfacción de las gestantes y de los y las profesionales. La investigación determinó que la infraestructura para brindar las consultas de control prenatal de las Áreas de Salud de la provincia de Heredia está en buen estado, sin embargo, requiere mantenimiento y una adecuación para que sean accesibles a toda la población. Además, la Caja Costarricense de Seguro Social tiene una baja cobertura de atención prenatal con cumplimiento de criterios de calidad y por otra parte el reporte de la información obtenida durante las consultas de control prenatal en el Carnet Perinatal, es incompleta y errónea. Finalmente hay escasa o casi nula participación de los y las profesionales en Enfermería Ginecológica, Obstétrica y Perinatal, en el proceso del control prenatal, a pesar de que la legislación nacional y estudios reconocen que estos y estas profesionales cuentan con las competencias necesarias para brindar un adecuado control.

Palabras clave: Control-prenatal, embarazo, evaluación, morbimoralidad-materna, profesionales-en-salud.

\footnotetext{
${ }^{1}$ Fecha de recepción: 23 de setiembre del 2013

Fecha de aceptación: 6 de marzo del 2014

${ }^{2}$ Enfermera Ginecobstétrica y Perinatal. Hospital Rafael A. Calderón Guardia. Correo electrónico: nathis03@gmail.com

${ }^{3}$ Enfermera Ginecobstétrica y Perinatal. Hospital México. Correo electrónico: grecamposv@gmail.com
} 


\title{
Analysis of prenatal care that is provided to pregnant women in the province of Heredia who give birth in the San Vicente de Paul Hospital ${ }^{1}$
}

Institution: San Vicente de Paúl Hospital

\author{
Nathalie Alfaro Vargas ${ }^{2}$ \\ Grettel Campos Vargas ${ }^{3}$
}

CITED

Alfaro, N. y Campos, G. (2014). Analysis of prenatal care that is provided to pregnant women in the province of Heredia who give birth in the San Vicente de Paul Hospital, Heredia, during 2012. Rev. Enfermería Actual en Costa Rica, 26, 1-19. Available:

<http://www.revenf.ucr.ac.cr/cuidado.pdf> ISSN 1409-4568

\begin{abstract}
This article presents the main results of a quantitative research design with a non- experimental descriptive cross, which aimed to analyze prenatal care that is provided to pregnant women in the province of Heredia who gave birth at St. Vincent Hospital de Paul in 2012. The population consisted of pregnant women who delivered at the hospital between the months of December 2011 to November 2012 and by medical professionals and nurses who work for the health areas of Heredia and San Vicente de Paul Hospital, which provide prenatal control. To collect information three instruments considered infrastructure, equipment and procedures to carry out prenatal care, in addition, the level of satisfaction of pregnant women and the professionals were used. The investigation determined that the infrastructure to provide the prenatal control of health areas in the province of Heredia is in good condition, however, requires maintenance and suitability to be accessible to the entire population. Furthermore, the Costa Rican Social Security has a low coverage of antenatal care with compliance with quality criteria and otherwise report the information obtained during the prenatal control in the Perinatal Carnet is incomplete and incorrect. Finally there is little or almost no participation of professionals and Gynecological Nursing, Obstetric and Perinatal, in the process of prenatal care , although national legislation and recognize that these studies and these professionals have the necessary skills to provide adequate control.
\end{abstract}

Keywords: evaluation, morbidity-and-mortality-maternal, prenatal-care, pregnancy, professional-healthcare.

\footnotetext{
${ }^{1}$ Date of receipt: september 23, 2013

Date of acceptance: march 6, 2014

${ }^{2}$ Gynecobstetric and Perinatal Nurse. Dr. Rafael A. Calderón Hospital. E-mail: nathis03@gmail.com

${ }^{3}$ Gynecobstetric and Perinatal Nurse. México Hospital. E-mail: grecamposv@gmail.com
} 


\section{Revista Electrónica Enfermería Actual en costa Rica}

\section{INTRODUCCIÓN}

El cuidado que se le brinda a las mujeres gestantes tiene como objetivo prevenir e identificar oportunamente problemas de salud en la madre y el bebé, esto se encuentra refrendado desde los organismos internacionales:

En septiembre del 2000, los Jefes de Estado y de Gobierno de 147 países y 42 ministros y jefes de delegación se reunieron en la Asamblea General de las Naciones Unidas para emprender la tarea de determinar cómo mancomunar sus voluntades y su compromiso de realizar un esfuerzo conjunto para revitalizar la cooperación internacional destinada a los países menos desarrollados y, en especial, a combatir decisivamente la pobreza extrema.(Naciones Unidas, 2005)

Es a partir de esta reunión que se generan los Objetivos de Desarrollo del Milenio (ODM), en ello se encuentran los relacionados con el mejoramiento de la salud donde el número 4 y 5 hablan respectivamente de: "Reducir la mortalidad infantil" y "Mejorar la salud materna".

Para el año 2005, mitad del periodo de plazo para alcanzar las metas propuestas, los índices de mortalidad infantil disminuyeron en la región, sin embargo, existen grandes diferencias entre los países, Costa Rica se encuentra dentro de los países con menores tasas de mortalidad. "Con respecto a la mortalidad materna a nivel de la región hay un estancamiento y únicamente Uruguay, Chile, Cuba, Santa Lucía, Argentina, Brasil y Costa Rica, aparece con niveles por debajo de 50 muertes por cada 100.000 nacimientos." (Naciones Unidas, 2005)

En Costa Rica la tasa de mortalidad infantil en 1990 fue de 15,3 y en el 2011 fue de 9,07 por cada mil nacimientos, esto demuestra que ha disminuido según lo pactado en los ODM. De acuerdo con el informe del Ministerio de Salud, para el 2011 la provincia de Limón obtuvo la tasa de mortalidad infantil más alta, de 10,54 muertes por 1.000 nacidos vivos y la provincia de Heredia presentó la menor tasa de 7,57.

Con respecto a la tasa de mortalidad materna, entre los años 2000 y 2011 se muestra una variabilidad caracterizada por altibajos, en el 2000 la tasa fue de 3,6 por cada 10.000 nacimientos, en el 2010 fue de 3,93, la mayor registrada, y en el 20071,90 , la menor registrada y ya para el 2011 la tasa fue de 2,11 por cada 10.000 nacimientos. (Ministerio de Salud, 2011)

Los datos por provincia revelan que Limón obtuvo la mayor tasa de mortalidad, 6,20 por cada 10.000 nacimientos, seguida de Alajuela con 4,75; en las provincias de Cartago, Guanacaste, Puntarenas y Heredia no ocurrió ninguna muerte materna. (Ministerio de Salud, 2011).

En este punto es importante destacar que el nuevo Hospital San Vicente de Paúl se inauguró el 04 de mayo del 2010 y el traslado de las personas usuarias inició el 15 de julio de ese mismo año, razón por la cual se refería a las gestantes con algún riesgo en la atención de su parto o gestación al Hospital México, situación que provocó un subregistro de datos.

Continuando con la mortalidad materna en la provincia de Heredia para el 2012 en el Hospital San Vicente de Paúl se reportan 3 muertes maternas según la Comisión de Mortalidad Materna e Infantil de dicho hospital, aspecto que refleja la situación nacional para ese mismo año, ya que la mortalidad materna llegó a 22 muertes, 


\section{Revista Electrónica Enfermería Actual en costa Rica}

aumentando esta cifra con respecto al año 2011 que fue de 15 muertes maternas, según datos preliminares proporcionados por la Comisión Nacional de Mortalidad Materna e Infantil.

Respecto de lo anterior Ledesma (2011) menciona que la primera causa de mortalidad materna es la ineficiencia en el control prenatal, seguido de complicaciones en el trabajo de parto y el puerperio, mala identificación de diagnósticos y ausencia de la consulta preconcepcional.

Con respecto a la ineficiencia del control prenatal Ledesma (2011) refiere que se debe principalmente por el retraso en la captación, las dificultades de acceso, la no aplicación de las normas y el retardo en la definición del riesgo.

El control prenatal al ser un conjunto de acciones y procedimientos sistemáticos y periódicos, destinados a la prevención, diagnóstico y tratamiento de los factores que puedan condicionar morbimortalidad materna y perinatal, permite evaluar la evolución del embarazo y preparar a la madre para el parto y la crianza de su hijo o hija (CCSS, 2009). De esa forma, se puede controlar el momento de mayor morbimortalidad en la vida del ser humano, como es el período perinatal y la principal causa de muerte de la mujer joven como es la mortalidad materna.

Tamayo y Ahued (2003) mencionan al respecto que el control prenatal reduce la morbimortalidad materna y perinatal, el número de partos prematuros y el de productos con bajo peso al nacer; de igual forma permite identificar factores de riesgo, lo cual hace posible establecer acciones preventivas y terapéuticas oportunas durante el embarazo.

Es fundamental tener claro que en el control prenatal es importante no sólo el número de visitas que la gestante realice al centro de salud, sino cuándo fueron realizadas estas durante el transcurso del embarazo y con qué calidad. El control prenatal, con inicio desde el primer mes de embarazo y con consultas médicas una vez por mes hasta el parto, permite identificar complicaciones del embarazo como infecciones cervicovaginales, diabetes gestacional, preeclampsia, infecciones sistémicas (VIH y otras), además, permite establecer medidas preventivas oportunas de acuerdo con la situación de la embarazada en el momento oportuno, lo que significa un costo menor en su atención posterior y mejora la calidad de la atención y la calidad de vida de las gestantes y sus hijos.

El enfoque de riesgo es un instrumento para identificar los problemas prioritarios que contribuyen a la mortalidad perinatal en los diferentes niveles de atención y cobertura, a partir de esto permite crear y distribuir en forma racional los recursos que se requieran, proponiendo estrategias que muestren beneficios en la población obstétrica, así como en la disminución de la mortalidad perinatal, por esto el control prenatal debe incluir este enfoque. (Vilchis, Gallardo, Rivera y Ahued, 2002)

\section{Asimismo según Sánchez, Pérez, Pérez, y Vásquez (2005),}

refieren que el control prenatal adecuado (más de cinco consultas con inicio en el primer trimestre del embarazo) impacta en la prevención del síndrome de insuficiencia respiratoria en el neonato, el bajo peso al nacimiento y la prematurez, ya que evitarla es uno de los propósitos del control prenatal.(p.379) 
Consecuentemente, se mejora la calidad de la atención al conocer el estado de salud de la gestante con anticipación y así mejora la calidad de vida para el binomio madre-hijo, aún en madres adolescentes a quienes se logra referir para apoyo psicológico y terapia familiar. Por otro lado, la falta de oportunidad y continuidad de la atención prenatal conlleva a partos distócicos que culminan en cesáreas y aumentan los costos de la atención del parto y del recién nacido (Tamayo y Ahued, 2003), de ahí la importancia de que las acciones que se realicen en el control prenatal sean congruentes con las necesidades de la población y además que sean previamente planificadas por los y las profesionales de salud.

En Costa Rica, la CCSS publicó en el 2009 la Guía de Atención Integral a las Mujeres Niños y Niñas en el periodo prenatal, parto y posparto, la cual está basada en los principios y el modelo de atención prenatal de la Organización Mundial de la Salud (OMS), esta constituye la principal guía para los y las profesionales que brindan el control prenatal.

Para la evaluación del control prenatal que se brinda en el país, existen únicamente los Compromisos de Gestión de la CCSS, los cuales surgen en 1996 a partir de una reforma sanitaria impulsada por dicha institución con el objetivo de aumentar la calidad y eficiencia de los servicios de salud y contener la expansión del gasto. Los Compromisos de Gestión son un instrumento que vincula las metas de las actividades de los proveedores de salud con la asignación de presupuesto por parte de la administración central de la CCSS. (Cercone, Durán y Muñoz, $\underline{2000)}$

Respecto al control prenatal, los Compromisos de Gestión realizan una evaluación cuantificable principalmente de la cobertura y de algunos criterios de calidad como captación temprana y concentración. Según el Área de Estadística de la CCSS, las cifras de los Compromisos de Gestión revelan que durante el 2005 a nivel nacional se dio un total en cobertura y calidad de 56,41\%, fue el último año donde se cita la calidad dentro de la estadística; la cobertura lograda fue de $79,18 \%$ y la pactada de $72,26 \%$, para un total de 77.539 embarazadas en ese año.

Continuando con los datos obtenidos de la evaluación del compromiso de gestión hasta el 2009, en el 2006 la población meta fue de 77.377 gestantes, la cobertura con cumplimiento de criterios de 63,01\%. Para el 2007 la población meta fue de 77.124 embarazadas, la cobertura con cumplimientos de criterios de 64\%. En el 2008 la población meta fue de 77.682 gestantes y la cobertura con cumplimiento de criterios fue de $76 \%$, como se observa aumentó con respecto a los dos años anteriores, sin embargo para el año 2009 disminuyó ya que la población meta fue de 70.302 embarazadas y la cobertura con cumplimiento de criterios de 74,93\% para un total de 63.910 nacimientos.

Particularmente en la región de Heredia la cobertura en el 2005 fue de 79,6\% y la calidad de un 54,33\%, para los años del 2006 al 2009 la cobertura con cumplimiento de criterios fue de 58,32\%; 63,63\%; 77,73\% y 75,88\% respectivamente. (Cercone, Durán \& Muñoz, 2000).

Además de los resultados de los Compromisos de Gestión, existen a nivel nacional tres investigaciones que evalúan aspectos relacionados con el control prenatal, una realizada Servicio de Obstetricia del Hospital de las Mujeres Dr. Adolfo Carit Eva (2002) la cual tuvo como objetivo identificar algunos resultados perinatales en el Hospital, con relación al lugar en el cual las gestantes recibieron el control prenatal, en esta se concluye que el 


\section{Revista Electrónica Enfermería Actual en costa Rica}

control prenatal es altamente indiferenciado entre establecimientos de la CCSS, sin embargo, es necesario que documenten las acciones para lograr resultados perinatales más satisfactorios.

Por su parte Leal \& Barboza (2008) realizaron una caracterización del control prenatal y parto de la población inmigrante atendida en el Hospital Dr. Rafael Ángel Calderón Guardia en comparación con la población nacional, encontraron que 1 de cada 5 gestantes es inmigrante principalmente de nacionalidad nicaragüense, aproximadamente la mitad de estas presentan un control prenatal tardío e incompleto, sin embargo concluyen que la morbilidad previa e inducida por el embarazo y los resultados perinatales no difiere entre ambas poblaciones.

Finalmente, en Heredia, Arias, Jiménez, Granados, Montero \& Calvo (2000) evaluaron el nivel de satisfacción y de educación respecto al embarazo en 179 gestantes que asistieron al control prenatal en el EBAIS de San Pablo, el 76\% refirió que los servicios brindados fueron adecuados y las gestantes evidenciaron conocimientos suficientes en cuanto a signos de embarazo y síntomas de complicaciones obstétricas.

Como se observa existen iniciativas de evaluar la calidad del control prenatal a nivel nacional, sin embargo, se evidencia la necesidad de desarrollar investigaciones que permitan analizar el proceso del control prenatal y todo lo que este engloba, a las gestantes, a los y las profesionales en salud que brindan los servicios, los recursos y materiales, entre otros; para así obtener resultados que sirvan de evidencia para continuar con las acciones que favorezcan a la población o bien modificarlas a favor de la calidad de la atención en salud.

Por estas razones se planteó como objetivo de investigación analizar el control prenatal que se brinda a las gestantes de la provincia de Heredia que tienen su parto en el Hospital San Vicente de Paúl durante el año 2012.

\section{MATERIALES Y MÉTODOS}

El enfoque de la investigación es cuantitativo, con un diseño no experimental de tipo descriptivo transversal. (Hernández, Fernández y Baptista, 2006). La población estuvo constituida por 5.014 gestantes que tuvieron su parto en el Hospital San Vicente de Paúl, Heredia entre los meses de diciembre 2011 a noviembre 2012, además la población de profesionales en medicina 60 que laboran para las Áreas de Salud de Heredia y el Hospital, que brindan control prenatal.

Se aplicó un muestreo probabilístico, por lo que la muestra estuvo constituida finalmente por 371 gestantes, (Hernández y otros, 2006) Además, para determinar la muestra de las Áreas de Salud y de los y las profesionales de salud que brindan el control prenatal se realizó un muestreo por conglomerados (Kish, 1979), para el cual se hizo en primer lugar una selección aleatoria de conglomerados en la que de las 11 Áreas de Salud, se seleccionaron 5 más el Hospital, posteriormente se realizó un submuestreo en el cual resultaron seleccionados los siguientes 15 sectores o EBAIS: Barva 1 y 2, San Roque, Santo Domingo, San Vicente Este y Oeste, San Pablo Centro, Quintana, La Amalia, San Joaquín, Llorente 2, Barreal y Aurora1 y 2. Se seleccionó a un profesional de la salud por sector, además de tres profesionales del Hospital.

Para realizar el análisis del control prenatal que se brinda a las gestantes de la provincia de Heredia que tienen su parto en el Hospital San Vicente de Paúl durante el año 2012, se establecieron cinco objetivos, (1) 
identificar el plan del control prenatal en Costa Rica; (2) describir el funcionamiento del control prenatal, en términos de infraestructura, equipamiento, procedimiento y cobertura; (3) identificar los criterios utilizados para evaluar el control prenatal en Costa Rica; (4) describir la percepción que tienen las gestantes así como el o la profesional de salud sobre el control prenatal que se brinda; y (5) identificar el papel que desempeña el profesional de Enfermería Ginecológica Obstétrica y Perinatal en el control prenatal.

Los instrumentos utilizados fueron el cuestionario dirigido a las mujeres de la provincia de Heredia que tienen su parto en el Hospital San Vicente de Paúl aplicado durante su hospitalización, el instrumento para valorar la infraestructura y el equipamiento con el que cuentan las Áreas de Salud de la provincia de Heredia para brindar la consulta de control prenatal y una entrevista semiestructurada dirigida a los y las profesionales de la salud que brindan control prenatal en las Áreas de Salud de la provincia de Heredia aplicado durante la visita a los Areas de Salud.

Los datos obtenidos, se transcribieron y ordenaron mediante el uso del programa Microsoft Excel, estos fueron presentados gráficamente por medio de tablas y gráficos, según sus características. Posterior a esto, se interpretaron y analizaron, para lo cual fue necesario contrastarlos con la normativa costarricense y con la teoría relacionada con el proceso del control prenatal.

\section{Consideraciones bioéticas}

Las personas que participaron de esta investigación lo hicieron de manera voluntaria previo consentimiento informado, se mantuvo el anonimato y la descontinuación de su participación no representó consecuencias negativas. Cabe destacar que el protocolo de la investigación fue avalado por el Comité Local de Bioética en Investigación del Hospital San Vicente de Paúl en la sesión 003-2012, celebrado el 03 de mayo del 2012 .

\section{RESULTADOS}

A continuación, se presentan los resultados según los objetivos mencionados en el apartado anterior:

1. Plan del control prenatal en Costa Rica.

La guía de Planificación Local emitada por la Caja Costarricense de Seguro Social (2009) para la atención integral a las mujeres, niños y niñas en el periodo prenatal, parto y posparto, se divide en dos actividades: 1 . Análisis del diagnóstico de salud o ASIS local y 2. Programación Local basada en el punto 1. Debido a lo anterior, no fue posible determinar en esta investigación un porcentaje de cobertura esperado debido a la diversidad de características que presenta cada área de salud.

2. Funcionamiento del control prenatal, en términos de infraestructura, equipamiento, procedimiento y cobertura.

Con relación a la infraestructura se realizó una observación en cinco Áreas de Salud y el hospital, en total se observaron 15 consultorios donde se brinda el control prenatal; tres de las Áreas cuentan con un consultorio por 


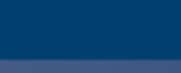

(c)

\section{Revista Electrónica Enfermeria actual en costa Rica}

\section{www.revenf.ucr.ac.cr}

EBAIS para brindar estas consultas en las dos restantes algunos EBAIS cuentan con dos o tres consultorios; en el Hospital cuentan sólo con tres.

Se encontró que en las Área de Salud de Santo Domingo y en el hospital el estado de la infraestructura de los consultorios es bueno, en las Áreas de Salud Barva, San Pablo y Virilla es regular y en el Área de Salud de Belén Flores es malo. Al evaluar las condiciones que establece la Ley 7600 Igualdad de oportunidades para las personas con discapacidad; para procurar la facilidad de acceso al espacio físico, únicamente en ocho de los consultorios se cumplía con ésta, en el caso de los siete consultorios restantes, el espacio era reducido y los accesos al consultorio no permitían la entrada de una silla de ruedas.

Respecto del equipamiento, en cinco de los consultorios, los pertenecientes al hospital y al Área de Salud de Belén Flores, se encontraba completo, según lo establecido por la CCSS, en los consultorios de las demás áreas no se contaba con lugol; con relación al equipo de parto en los EBAIS de Llorente, San Joaquín de Flores y Barva este equipo estaba disponible en emergencias, particularmente en el Área de Barva estos equipos se encontraban vencidos. El estado del equipo en general era bueno pero requería de mantenimiento, lo cual coincide con lo referido por los y las profesionales de la salud entrevistados que brindan control prenatal; sólo en uno de los consultorios el equipo se encontró en mal estado.

El control prenatal fue brindado por el médico general en un $86,5 \%$, el $13 \%$ por especialista en ginecobstetricia y un $0,3 \%$ enfermería ginecobstétrica. Es decir la participación de enfermería es casi nula y al realizar las visitas a las Áreas de Salud, se determinó que aunque en algunas habían enfermeras y enfermeros ginecobstétricos, estos ocupaban cargos en enfermería general y que las únicas acciones que realizaban relacionadas con el control prenatal son la preconsulta y el registro de la cantidad de gestantes que asisten al control.

En relación al procedimiento del control prenatal que recibieron las mujeres que tuvieron su parto en el Hospital San Vicente de Paúl, Heredia, 2012, en la tabla 1 se presentan los resultados generales acerca de las actividades realizadas durante las consultas de control prenatal. 


\section{Tabla 1}

Hospital San Vicente de Paúl: Distribución de la opinión de las mujeres respecto a las actividades realizadas en las consultas de control prenatal, 2012

(Frecuencias absolutas)

\begin{tabular}{l|c|c|c|c|c|c|c|c|c}
\hline $\begin{array}{c}\text { Área de } \\
\text { Salud }\end{array}$ & $\begin{array}{c}\text { Número } \\
\text { de } \\
\text { mujeres }\end{array}$ & $\begin{array}{c}\text { Presencia } \\
\text { de } \\
\text { sangrado } \\
\text { vaginal }\end{array}$ & $\begin{array}{c}\text { Presencia } \\
\text { de fiebre }\end{array}$ & $\begin{array}{c}\text { Presencia } \\
\text { de } \\
\text { hidrorrea }\end{array}$ & $\begin{array}{c}\text { Presencia de } \\
\text { movimientos } \\
\text { fetales }\end{array}$ & $\begin{array}{c}\text { Presencia } \\
\text { de } \\
\text { cefalea }\end{array}$ & $\begin{array}{c}\text { Presencia } \\
\text { de } \\
\text { acúfenos }\end{array}$ & $\begin{array}{c}\text { Presencia } \\
\text { de edema }\end{array}$ & $\begin{array}{c}\text { Presencia } \\
\text { de dolor } \\
\text { pélvico }\end{array}$ \\
\hline $\begin{array}{l}\text { Belén } \\
\text { Flores }\end{array}$ & 35 & 31 & 16 & 22 & 35 & 10 & 5 & 24 & 23 \\
\hline Cubujuquí & 48 & 45 & 27 & 37 & 48 & 27 & 13 & 27 & 32 \\
\hline Barva & 25 & 24 & 12 & 21 & 25 & 14 & 8 & 21 & 20 \\
\hline San Isidro & 12 & 11 & 3 & 10 & 12 & 2 & 1 & 6 & 4 \\
\hline San pablo & 13 & 12 & 7 & 13 & 13 & 7 & 0 & 7 & 9 \\
\hline $\begin{array}{l}\text { San } \\
\text { Rafael }\end{array}$ & 32 & 27 & 16 & 21 & 32 & 17 & 9 & 22 & 18 \\
\hline $\begin{array}{l}\text { Santa } \\
\text { Bárbara }\end{array}$ & 20 & 18 & 15 & 17 & 20 & 12 & 7 & 12 & 15 \\
\hline $\begin{array}{l}\text { Santo } \\
\text { Domingo }\end{array}$ & 16 & 15 & 8 & 13 & 16 & 5 & 5 & 8 & 10 \\
\hline Horquetas & 8 & 8 & 6 & 7 & 8 & 3 & 2 & 4 & 6 \\
\hline $\begin{array}{l}\text { Puerto } \\
\text { Viejo }\end{array}$ & 39 & 35 & 25 & 32 & 39 & 21 & 17 & 25 & 28 \\
\hline Virilla & 49 & 46 & 26 & 34 & 48 & 20 & 15 & 24 & 23 \\
\hline HSVP & 43 & 40 & 24 & 40 & 43 & 23 & 20 & 30 & 33 \\
\hline $\begin{array}{l}\text { Otras } \\
\text { Áreas }\end{array}$ & 31 & 27 & 13 & 23 & 30 & 12 & 7 & 14 & 19 \\
\hline Totales & 371 & 339 & 198 & 290 & 368 & 173 & 111 & 224 & 240 \\
\hline $\begin{array}{l}\text { \% en } \\
\text { relación al } \\
\text { total }\end{array}$ & $100 \%$ & $91,3 \%$ & $53,4 \%$ & $78,2 \%$ & $99 \%$ & $46,6 \%$ & $30 \%$ & $60,4 \%$ & $64,7 \%$ \\
\hline & & & & & & & & \\
\hline
\end{tabular}

A continuación se presentan los resultados detallados por cada uno de las acciones que conforman el procedimiento del control prenatal según lo establecidos en la Guía de la CCSS (2009):

\section{Entrevista con enfoque de riesgo:}

Con respecto a las preguntas realizadas por los y las profesionales de la salud durante el control prenatal a las gestantes entrevistadas, se encontró que las preguntas realizadas con mayor frecuencia fueron: la percepción de movimientos fetales, presencia de sangrado vaginal, presencia de hidrorrea y dolor en la parte baja del vientre, en orden descendente.

En relación a la indagación de signos neurohipertensivos los y las profesionales de la salud preguntaron en la mayoría de los casos si la gestante había presentado edema podálico en comparación con otros signos como la 


\section{Revista Electrónica Enfermería Actual en costa Rica}

cefalea y la presencia de acúfenos. Lo anterior a pesar de que el edema de miembros inferiores lo presenta el $80 \%$ de las gestantes, por lo tanto no es indicativo de severidad, únicamente si éste compromete las extremidades superiores y la cara (CEDIP, 2011)

Únicamente el $20 \%$ de las gestantes refirió que le habían realizado una entrevista con enfoque de riesgo completa, es decir los profesionales indagaron acerca de presencia de signos neurohipertensivos, dolor pélvico, hidrorrea, sangrado transvaginal, fiebre y percepción de movimientos fetales; algunas de las gestantes refirieron que en algunas ocasiones ellas tenían que preguntarle a los y las profesionales si algunos de los síntomas que presentaban eran normales o no, ya que por lo general ellos y ellas no les realizaban las preguntas. Lo cual va en detrimento de una buena consulta prenatal con enfoque de riesgo ya que no se profundiza en la información que puede ser relevante para la toma de decisiones en la atención de la gestante.

\section{Valoración}

Respecto de la valoración realizada por los y las profesionales de la salud, a la totalidad de la población de gestantes se le tomó la presión arterial, al 99\% de la población se le auscultó la frecuencia cardiaca fetal y se pesó y al $96 \%$ se le midió la altura uterina. El procedimiento menos realizado fue la revisión de extremidades inferiores para la detección de edema, el cual solo se le realizó al 46\% de las gestantes.

\section{Prescripción de tratamiento y pruebas de laboratorio}

Al 98\% de la población se le prescribió hierro y ácido fólico durante el control prenatal, el 70,4\% de las gestantes refieren que le prescribieron la vacuna contra el tétano, sin embargo, en la Historia Clínica Perinatal no se encontró registrada la aplicación de la vacuna.

En cuanto a la prescripción de pruebas de laboratorio, el 100\% de la población refirió que le habían prescrito análisis de sangre; con respecto al análisis de orina solo un 1,2\% refirió que nunca se le prescribió dicha prueba durante el control prenatal. El 55\% de las gestantes fueron referidas a otro servicio de salud durante el control prenatal, el servicio al que fueron referidas en mayor proporción (86\%) fue al de Odontología.

\section{Información}

La información brindada por los y las profesionales de salud durante el control prenatal, acerca de la actividad física y alimentación durante el embarazo, cuidados del recién nacido y métodos anticonceptivos seguros y efectivos que se pueden utilizar en el posparto y que no afectan la lactancia, se encontró que sólo el 6\% de las gestantes recibió información acerca de todos los temas y el 55\% recibió al menos información de uno de los temas, el $38 \%$ de las gestantes no recibió información acerca de ninguno de estos.

El tema que fue más abordado correspondió al de cuidados del recién nacido, según refirieron las gestantes les hablaron sobre la importancia del tamizaje y de la lactancia materna para el recién nacido; el tema menos abordado fue el de actividad física. 
Durante la entrevista algunas de las gestantes mencionaron que durante las consultas de control prenatal no se les suministraba esta información porque era en el curso de preparación para el parto donde se asumían estos temas, sin embargo, solo el 52\% de las gestantes afirmó recibir información acerca de la hora, fecha y lugar donde se iba a brindar el curso, del 48\% de mujeres que afirmó no recibir información acerca del curso, el 37\% se encontraba en el grupo de gestantes que no había recibido información acerca de ninguno de los temas antes mencionados.

\section{Llenado del Carnet Perinatal}

El 93\% de las gestantes portaban el Carnet Perinatal, al realizar la revisión de estos se encontró que sólo el $4 \%$ se encontraban completos y únicamente el $12 \%$ tenía información llena de la forma correcta.

En cuanto a los datos personales el 6,1\% de los carnets no los tenía reportados y del 93,9\% que sí los tenía reportados el $10,1 \%$ eran ilegibles ya que los mismos habían sido transferidos al carnet por medio de papel carbón. Además, el 93,9\% de los carnets tenían completa la información de los antecedentes patológicos familiares y personales de la gestante, sin embargo, el 9,3\% de estos eran ilegibles.

En cuanto a los antecedentes ginecobstétricos el 92,8\% de estos fueron reportados en el Carnet Perinatal, sin embargo, el 60,3\% de estos fueron reportados de manera errónea ya que incluían el embarazo actual dentro del número de gestas. Además, en el $2 \%$ de los casos no se incluyeron los datos del hijo o hija anterior. El riesgo obstétrico se reportó en el $51,3 \%$ de los casos, los resultados de laboratorios en el 14,8\% y la gráfica de peso en el $13 \%$.

Respecto al reporte de los datos del embarazo actual en el Carnet Perinatal, se encontró que los datos menos reportados fueron los resultados del PAP (55,7\%) y del examen físico $(76,5 \%)$, la fecha de la última menstruación y la fecha probable del parto fue reportada en el 94,8\% de los casos y la medición de la altura uterina se reportó en el $98,6 \%$.

Por medio de la revisión del Carnet Perinatal se indagó también acerca de sí las gestantes se captaron tempranamente, es decir, sí habían tenido su primer control prenatal en el primer trimestre de gestación, el $87 \%$ de las gestantes se captó tempranamente, Además, se determinó que el control prenatal fue periódico, ya que la captación fue temprana (durante el primer trimestre) en el 80,6\% y el 87,8\% de las gestantes acudió a más de ocho citas del control prenatal, según lo establece la CCSS (2009) para los embarazos de bajo riesgo se espera que las gestantes acudan a 5 o más consultas de control prenatal.

\section{Criterios utilizados para evaluar el control prenatal en Costa Rica.}

El último dato con el que se cuenta para la provincia de Heredia es el del año 2009, en este año particularmente en la región de Heredia la población meta fue de 70.302 gestantes, se reportaron 63.910 nacimientos y la cobertura de atención prenatal con cumplimiento de criterios de calidad fue de 74,93\%.

Percepción de las gestantes y de los y las profesionales de salud sobre el control prenatal que se brinda. 
Se indagó si las consultas de control prenatal fueron gratuitas para lo cual el $99,7 \%$ de las gestantes refirieron que sí, la persona que indicó que no lo recibió gratuitamente se refirió a la rebaja mensual de su salario correspondiente al seguro; si la asistencia se realizó de manera voluntaria donde en el $98 \%$ si lo fue, el restante $2 \%$ refirió que asistió al control prenatal en una institución de la CCSS porque necesitaba tramitar la licencia por maternidad ya que en el centro privado donde llevaba su control prenatal no la tramitaba.

El 74\% de las gestantes refirió que durante las consultas de control prenatal se le solicitó autorización para llevar a cabo los diferentes procedimientos y pruebas de laboratorio, el $26 \%$ restante dijo que no se les informó y por lo tanto no se les pidió su consentimiento para realizarles la prueba para la detección del VIH, lo cual no cumple con lo establecido en la normativa: "Obtener el "Consentimiento" de la mujer para que se realice la prueba de ELISA -VIH.” (CCSS, 2009, p.46); el 97,8\% manifestó que se respetó su autonomía durante el control prenatal, el 2,2\% refirieron que se sintieron obligadas de algún modo a cumplir el tratamiento y las citas; $87,3 \%$ de las gestantes refirieron sentirse cómodas durante el control, el $12,7 \%$ refirieron que no por el trato recibido durante las citas y finalmente el $96 \%$ de las gestantes refirió que su privacidad fue respectada y el $4 \%$ restante mencionó que había sido examinada y entrevistada por más de una persona en una misma cita de control, lo cual les pareció incómodo.

Respecto del trato brindado por los y las profesionales de la Salud durante el control prenatal, el 39\% de las gestantes refirió que fue bueno, el 23\% muy bueno, el 18\% excelente, el 16\% regular y el $4 \%$ refirió que fue deficiente.

Las gestantes que refirieron que el control fue regular y deficiente, mencionaron que dieron esta calificación porque la persona que les brindó el control prenatal no atendía sus necesidades, por ejemplo que no las referían a ultrasonido, que no le explicaban algunos signos y síntomas que presentaban o bien que la forma de dirigirse a ellas no era la adecuada.

En cuanto a la percepción de los y las profesionales en salud que brindan control prenatal estos refirieron, al respecto de los recursos con los que cuentan, 12 de ellos mencionaron que la planta física es adecuada, los tres restantes consideraron que el espacio físico es reducido y la construcción antigua.

Respecto al equipo 11 de los y las profesionales refieren estar a gusto, los otros cuatro mencionaron que es necesario el mantenimiento y actualización de equipos y materiales, por otra parte dentro de los aspectos que refieren como puntos de mejora se destaca principalmente el mantenimiento del equipo Doppler, liberación en muestras de laboratorio, mejoras en el acceso a ultrasonido obstétrico, kit de toma de muestra de estreptococo y mejoras a la planta física.

Finalmente en relación al recurso humano 13 profesionales refieren que se necesita de profesionales en Enfermería Ginecológica Obstétrica y Perinatal para que apoyen el proceso del control prenatal y dos expresaron que es importante un profesional en Medicina Obstétrica y Ginecológica disponible en el I Nivel de Atención. Papel que desempeña el profesional de Enfermería Ginecológica Obstétrica y Perinatal en el control
prenatal. Papel que desempeña el profesional de Enfermería Ginecológica Obstétrica y Perinatal en el control
prenatal. 
Pese a esto la inclusión de Enfermería Ginecoobstétrica y perinatal ha sido casi nula, como lo revelan los resultados de esta investigación, ya que, como se mencionó anteriormente, el 86,5\% de las gestantes recibió el control prenatal por parte de profesionales en medicina general, el $13 \%$ por especialistas en medicina ginecobstétrica y únicamente el 0,3\% (1 persona) por profesionales en enfermería ginecobstétrica.

Cuando se le consultó a los y las profesionales que brindan el control prenatal en la provincia de Heredia acerca de cuál consideran que es el personal de Salud que se requiere para brindar un adecuado control prenatal, trece de los quince profesionales mencionaron dentro de sus respuestas a los profesionales en enfermería obstétrica, entre las razones que mencionaron están que poseen facilidad para desarrollar programas educativos y para preparar a las gestantes para el parto; son fundamentales para mejorar la captación y para brindar seguimiento en la comunidad y que poseen capacidad para realizar una buena valoración y referir al especialista en medicina según necesidad.

Una de las profesionales entrevistadas mencionó que ella considera que deberían ser los profesionales en enfermería obstétrica los que brinden el control prenatal, pero que ve como único inconveniente el hecho de que no pueden recetar fármacos por lo que siempre se requiere la participación de un profesional en medicina, sin embargo, en el decreto y en la Guía antes mencionada se establece que los y las profesionales en enfermería ginecobstétrica pueden prescribir hierro y ácido fólico, que son los fármacos recetados en el control prenatal.

\section{DISCUSIÓN}

En la Guía de Atención Integral a las Mujeres Niños y Niñas en el periodo prenatal, parto y posparto creada por la CCSS (2009), se resalta que el control prenatal debe ser equitativo, precoz, periódico, completo y con enfoque de riesgo; además, establece una pauta para los centros de salud en cuanto a cuales recursos técnicos, humanos y materiales son necesarios para brindar la atención prenatal. Además, establece las acciones que deben realizarse durante la consulta de control prenatal y menciona al personal de salud responsable de realizarlas.

La guía anterior, en el apartado de Planificación Local, refiere que para determinar la cobertura del control prenatal se deben realizar dos actividades, la primera es el análisis del diagnóstico de salud o ASIS local, el cual debe ser realizado en conjunto por la dirección del Área de Salud, el EBAIS y el personal administrativo, con el fin de determinar el número de embarazos esperados y las condiciones de la población que favorecen o no su acceso a una atención prenatal oportuna. La otra actividad es la programación local, que con base en la actividad anterior, se deben determinar las acciones que se van a realizar para incluirlas dentro de Plan Operativo Anual y el presupuesto, estas deben asegurar el acceso de la población y el cumplimiento de la normativa.

En Costa Rica el plan del control prenatal está amparado por el decreto No 35262-S "Norma Oficial Atención Integral a la Mujer durante el Embarazo de Bajo Riesgo Obstétrico", el cual fue publicado en La Gaceta en febrero del 2009, además, responde a los principios propuestos por la OMS (1985), los cuales mencionan que el control prenatal no debe ser medicalizado, debe estar basado en la mejor evidencia en cuanto al uso de tecnología y prácticas apropiadas, ser regionalizado y eficiente en la realización de referencias entre los distintos niveles de atención, ser multidisciplinario, integral, centrado en la familia gestante y sus necesidades y respectando los derechos de autonomía, privacidad, dignidad y confidencialidad. 
En ese sentido, es importante recordar que la CCSS se sustenta en los principios que propone la OMS (1985) para orientar la atención o control prenatal, dentro de estos se destaca que la atención prenatal no debe ser medicalizada, el cuidado fundamental debe ser provisto utilizando un conjunto básico de intervenciones necesarias y se debe aplicar la menor tecnología posible, además, debe utilizar la tecnología apropiada, un conjunto de acciones que incluyan métodos, procedimientos, tecnología, equipamiento y otras herramientas, todas aplicadas a resolver un problema específico. Estos principios pretenden reducir el uso de tecnología no necesaria, sin embargo, se espera que la infraestructura mínima sea completa, se encuentre en buen estado y disponible con el fin de cumplir el objetivo del control prenatal que corresponde a controlar la evolución del embarazo, preparar a la familia gestante para el parto y disminuir los riesgos en el proceso.

Es claro que la participación de profesionales en enfermería ginecológica, obstétrica y perinatal es de suma importancia para brindar a la población un servicio de calidad. Sin embargo, los resultados de este estudio dejan ver que la participación de éste profesional es prácticamente nula, a pesar de que en el decreto No $35262-\mathrm{S}$ y en la Guía de la CCSS (2009) se reconoce la importancia de la inclusión de éstos y además, el Colegio de Enfermeras y Enfermeros de Costa Rica establece en el Reglamento de Enfermería Ginecobstétrica y Perinatal de Costa Rica, que estos profesionales cuentan con las competencias cognitivas, técnicas y actitudinales que los convierten en el personal óptimo para liderar el control prenatal.

La CCSS (2009) menciona dentro de los objetivos del control prenatal la prevención del tétano materno y fetal, para esto establece un flujograma para guiar a los y las profesionales en la determinación de los casos donde debe aplicarse la vacuna, en los casos en los cuales la mujer no presenta el esquema de vacunas o este se encuentra dudoso la vacuna debe aplicarse, por lo tanto es importante que se registre en el carnet la condición de la mujer al respecto. Por otro lado, también se incluye la prescripción de pruebas de laboratorio para identificar la presencia de enfermedades maternas como la anemia, por lo que se implementó en el país a partir del año 1998 gracias al Decreto Ejecutivo $\mathrm{N}^{\circ}$ 27086-S, una suplementación de micronutrientes, el cual inició con el Plan Nacional de prevención de deficiencias de Micronutrientes, en el cual las mujeres en edad reproductiva y las gestantes son consideradas una población prioritaria, ya que dicha suplementación contribuye a disminuir la prevalencia de anemias nutricionales y de los defectos del tubo neural. (Ministerio de Salud, 1999) Otra de las pruebas de laboratorio que se estableció fue para detectar la posible incompatibilidad sanguínea materno-fetal, infecciones del tracto urinario, diabetes, tétano, sífilis y VIH.

Por otro lado, la CCSS (2007) estableció como meta para el año 2011 que el 65\% de gestantes recibieran atención odontológica preventiva, aunque no se encuentran datos acerca de la cobertura, con los datos obtenidos en esta investigación se puede inferir que la cobertura, al menos para la provincia de Heredia es baja, ya que solo el $49 \%$ de las gestantes fue referida a dicho servicio.

Respecto de la informaciòn que se brinda a las usuarias, se puede afirmar que en la mayoría de los casos no se cumple con el objetivo propuesto en la normativa de la CCSS, el cual establece que se debe brindar de forma continua información pertinente a la gestante y su familia que les permita mejorar la salud en forma integral, además, dicha información debe adaptarse a la edad, a la edad gestacional, al contexto, al lenguaje y a las necesidades. (CCSS, 2009) 
De igual manera se destaca que la educación en salud es uno de los objetivos del control prenatal, ya que le permite a la gestante y la familia la detección de signos de alarma y el fomento de factores protectores para la gestante y su bebé.

Un instrumento muy importante que brinda información es el llenado del Carnet Perinatal, el cual debe realizarse de forma completa y adecuada, ya que como lo menciona la normativa el objetivo de esta actividad es: "Dar seguimiento adecuado en la atención de las mujeres gestan-tes a través de proporcionarle a la usuaria una historia resumida de su embarazo, que pueda ser utilizada por ella y en diferentes establecimientos." (CCSS, 2009, p.49) Por lo tanto, en la mayoría de los casos no se cumplió con dicho objetivo, lo cual evidencia el desconocimiento de los y las profesionales acerca de cómo se debe completar este instrumento.

En Costa Rica el control prenatal es evaluado únicamente en el ámbito público, el instrumento utilizado es el 051091 "Cobertura de Atención Prenatal con criterios de calidad” incluido en el Compromiso de Gestión de la CCSS, los resultados de esta evaluación se presentan cada 5 años, según la decisión tomada por las autoridades de la CCSS desde el año 2004.

Dicho instrumento tiene como objetivo conocer la cobertura de atención prenatal con criterios de calidad. La cobertura la definen como el porcentaje de mujeres embarazadas que han sido atendidas por primera vez en el control prenatal en el año, utilizando los siguientes criterios de calidad (CCSS, 2003, p.35):

1. Captación temprana: primera consulta de control prenatal durante el primer Trimestre (primeras 13 semanas de gestación).

2. Concentración: como mínimo 2 consultas en el II trimestre (14-19 semanas y 20-25 semanas) y 2 consultas en el III trimestre (26-30 semanas y 31-40 semanas).

3. Historia clínica perinatal (CLAP) debidamente llena, la cual debe contener:

- Antecedentes personales, familiares y gineco-obstétricos: en la primera consulta.

- Datos del embarazo actual con llenado del cuadro de consultas: en cada consulta.

- Anotación de factores de riesgo y su abordaje.

4. Indicación e interpretación de pruebas de laboratorio (con abordaje de las alteradas), lo cual incluye:

- Hemoglobina y hematocrito: primera consulta y semanas 30-35.

- VDRL: primera consulta y semanas 30-35.

- Glicemia en ayunas y post prandial (o post carga): primera consulta y semanas 30-35.

- Examen general de orina: en cada consulta.

5. Prescripción de hierro y ácido fólico: debe cubrir toda la gestación y la prescripción debe realizarse en cada consulta de control.

La puntuación de cada uno de los componentes del indicador se realiza de la siguiente manera ( $\underline{\text { CCSS }}$ 2003, p.35 y 36):

- $\quad$ Numerador: número de mujeres que consultan por primera vez en el año durante su período de embarazo. El número de mujeres se toma de los datos registrados por el Departamento de Información y 


\section{Revista Electrónica Enfermería Actual en costa Rica}

Estadística de Salud, Informe Estadístico Mensual y Anual, Centro de Gestión Informática y del Sistema de Información en Salud (SIS).

- Denominador: total de embarazadas esperadas (se calcula con base en los nacimientos ocurridos el año anterior por 1.1). Los datos son proporcionados por la Dirección Actuarial y de Planificación Económica, con base en el Instituto Nacional de Estadística y Censo.

- $\quad$ Porcentaje de expedientes con criterio de calidad: muestra de expedientes que cumplan todos los criterios de calidad.

La fórmula para realizar el cálculo es la siguiente (CCSS, 2003, p.36):

Total de mujeres que consultan por primera vez durante el período de embarazo (primera vez en el año y primera vez en el embarazo) x 100. Total de embarazos esperados en el mismo período.

Finalmente, la CCSS (2003) define como cobertura de atención prenatal con criterios de calidad al porcentaje de expedientes que cumplieron todos los criterios de calidad, por el resultado de cobertura previo a la revisión de los expedientes, además, considera subcategorías del indicador por grupo de edad, los cuales son: menores de 20 años, de 20 a 34 años y mayores de 35 años.

Es importante destacar la importancia de la relación entre proveedores de salud y usuarias, ya que una buena relación favorece el acceso precoz, oportuno y continuo de la gestante hacia los servicios obstétricos mediante estrategias de comunicación eficaz, la mejora de la cordialidad, el trato, el respeto y la empatía hacia estas. Al mejorar esta relación, se facilita el desarrollo conjunto, de parte de los agentes locales de salud y la familia gestante, de acciones en pro de la salud de la mujer embarazada. (Seclen, Benavides, Jacoby, Velásquez y Watanabe, 2004)

Respecto de la infraestructura y equipamiento, es un aspecto básico dentro de la mejora de la calidad de los servicios obstétricos ya que repercuten en la atención de la gestante y por lo tanto en la calidad del servicio. De ahí que los y las profesionales consideran un que es un tema de gran importancia y aunque reconen que ha habido mejoría en la dotación de equipo y en infraestructura todavía se requiere mayor mantenimiento y actualización del equipo.

Un aspecto importante de tomar en cuenta para este estudio es la participación del Colegio de Enfermeras y Enfermeros de Costa Rica, que en conjunto con el Comité Salud de la Mujer y Perinatología crearon el Reglamento de Enfermería Ginecobstétrica y Perinatal de Costa Rica, en el año 2005, en este se establecen las competencias cognitivas, técnicas y actitudinales que deben tener los y las profesionales en esta disciplina para poder brindar una atención de calidad a la familia gestante, lo que incluye su participación dentro del proceso del control prenatal.

Entre las competencias se citan, poseer conocimiento acerca del proceso pregestacional, gestacional y posparto, derechos humanos integrando los derechos sexuales y los derechos reproductivos, manejo de emergencias obstétricas y estrategias de maternidad sin riesgo. Además, tener la capacidad de gestionar la 


\section{Revista Electrónica Enfermería Actual en costa Rica}

atención a la familia gestante, identificar e interpretar el riesgo, aplicar principios clínicos y éticos en la atención, y promover la salud de la familia gestante.

Partiendo de lo expuesto anteriormente se puede afirmar que los y las profesionales en Enfermería Ginecológica, Obstétrica y Perinatal constituyen un personal idóneo para brindar el control prenatal, esto ha sido reconocido en la legislación costarricense, ya que como se mencionó anteriormente, en el año 2009 se firmó el Decreto $\mathrm{N}^{\circ}$ 35262-S "Norma Oficial Atención Integral a la Mujer durante el Embarazo de Bajo Riesgo Obstétrico" en el cual se menciona que los y las profesionales en enfermería obstétrica deben participar en la atención de la consulta prenatal de las gestantes con bajo riesgo obstétrico en el primer nivel de atención, ya que esta participación asegura que la atención sea de calidad y equitativa.

Por otra parte en la Guía de Atención Integral a las Mujeres Niños y Niñas en el periodo prenatal, parto y posparto, publicada por la CCSS también en el año 2009, establece como responsables de las actividades de la consulta de control prenatal a los y las profesionales en enfermería ginecobstétrica junto con los y las profesionales en medicina general.

Como se puede notar según la percepción de la mayoría de los y las profesionales en Salud se requiere de profesionales en Enfermería Obstétrica y Perinatal que brinden control prenatal y trabajen activamente dentro del equipo de apoyo que asiste a las gestantes.

Sin embargo, en nuestro país desde que se realizaron las reformas del sector salud a partir de la década de los años 70 se ejecutaron programas para extender la cobertura en salud y con esto se dio la exclusión de las enfermeras generales y/u obstétricas, las cuales se encargaban de las consultas del programa de salud maternoinfantil, de las actividades de educación en salud, atención de los partos y además, de capacitar a las parteras de la zona, lo cual disminuyó considerablemente la mortalidad materno infantil. Por lo tanto a partir de ese momento el control prenatal en el primer nivel de atención es brindado por un profesional en medicina general y según el nivel de riesgo que presente la gestante se refiere la atención con un profesional en medicina ginecobstétrica.

Cabe destacar que hasta la fecha no existe ninguna iniciativa por parte de la CCSS ni del Ministerio de Salud para volver a incluir a los y las profesionales en enfermería ginecobstétrica en la atención prenatal, a pesar de la importancia del papel de estos y estas profesionales en el control prenatal y su competencia profesional está definida y sustentada científicamente.

\section{CONCLUSIONES}

En Costa Rica se cuenta con un plan de control prenatal bien definido, el cual considera la importancia de la inclusión de los y las profesionales en Enfermería Ginecológica, Obstétrica y Perinatal.

La infraestructura, así como el equipo para brindar las consultas de control prenatal de las Áreas de Salud de la provincia de Heredia se encuentran en buen estado, sin embargo, requieren de mantenimiento una adecuación para que sea accesible a toda la población. 
La CCSS por medio de las acciones realizadas tiene una baja cobertura de atención prenatal con cumplimiento de criterios de calidad.

A pesar de que existe cumplimiento de muchos aspectos y procedimientos que se realizan en la consulta prenatal, como la valoración física, prescripción de tratamiento y pruebas de laboratorio, entre otros, se debe mejorar la entrevista con enfoque de riesgo y brindar mayor información a las gestantes así como el llenado del carnet perinatal.

Es imprescindible la inclusión de los y las profesionales en Enfermería Ginecológica, Obstétrica y Perinatal dentro del proceso del control prenatal, dada la relevancia de su participación para mejorar los índices de morbilidad y mortalidad materno-fetal.

\section{REFERENCIAS BIBLIOGRAFICAS}

Arias, R., Jiménez, J., Granados, E., Montero, J. \& Calvo, C. (2000). Estudio de la satisfacción de las mujeres en edad reproductiva con el servicio prenatal en relación con el nivel de educación y el inicio del control prenatal en San Pablo de Heredia. Heredia: COOPESIBA. R.L.

Caja Costarricense del Seguro Social. (2003). Fichas técnicas 2004. Área de Salud Primer y Segundo Nivel de Atención. Costa Rica: C.C.S.S.

Caja Costarricense del Seguro Social. (2007). Atención odontológica preventiva en el primer nivel de atención. Costa Rica: C.C.S.S.

Caja Costarricense del Seguro Social. (2009). Guía de Atención Integral a las Mujeres Niños y Niñas en el periodo prenatal, parto y posparto. Costa Rica: C.C.S.S.

CEDIP. (2011). Síndromes hipertensivos del embarazo. Boletín Perinatal, 1(2).

Cercone, J., Durán, F. \& Muñoz, G. (2000). Compromisos de Gestión, Rendición de Cuentas y Corrupción en los Hospitales de la Caja Costarricense de Seguro Social. Banco Interamericano de Desarrollo.

Colegio de Enfermeras y Enfermeros de Costa Rica. (2005). Reglamento de Enfermería Ginecobstétrica y Perinatal de Costa Rica. Costa Rica: La Gaceta \#10, 14 Enero 2005.

El Presidente de la República y la Ministra de Salud. (2009). Decreto No 35262-S. Costa Rica: La Gaceta 29 Mayo 2009.

Hernández, R., Fernández, C. \& Baptista, P. (2006). Metodología de la Investigación. 4ta Edición. México: McGraw-Hill Interamericana.

Kish, L. (1979). Muestreo de encuestas. México: Editorial Trillas. 
Leal, M. \& Barboza, J. (2008). Características del control prenatal y el parto de las mujeres inmigrantes atendidas en el Hospital Dr. Rafael Ángel Calderón Guardia. Revista Acta Médica Costarricense, 50 (2), 107-111.

Ledesma, L. (2011). Estrategia integral para la reducción de la mortalidad materna. Costa Rica: Ministerio de Salud.

Ministerio de Salud. (1999). Plan Nacional para la Prevención de Deficiencias de Micronutrientes 1999-2002. Costa Rica.: Ministerio de Salud.

Ministerio de Salud. (2011). Memoria Institucional 2011. Capítulo IV: Análisis y Determinantes Sociales de la Situación de Salud. Costa Rica: Ministerio de Salud de Costa Rica.

Naciones Unidas. (2005). Objetivos del Desarrollo del Milenio. Una mirada desde América Latina y el Caribe. Naciones Unidas.

Sánchez, H; Pérez, G; Pérez, P \& Vásquez, F (2005). Impacto del control prenatal en la morbilidad y mortalidad neonatal. Revista Instituto Mexicano del Seguro Social, 43 (5), 377-380.

Seclen, J., Benavides, B., Jacoby, E., Velázquez, A. \& Watanabe, E. (2004). ¿Existe una relación entre los programas de mejora de la calidad y la satisfacción de usuarias de atención prenatal?: experiencia en hospitales del Perú. Rev Panam Salud Pública, 16(3), 149-57.

Tamayo, C \& Ahued, J. (2003). Recomendaciones generales para mejorar la calidad de la atención obstétrica. México. Comisión Nacional de Arbitraje Médico, 71, 409-420.

Vilchis, P; Gallardo, J; Rivera, M \& Ahued, J. (2002) Mortalidad perinatal: una propuesta de análisis. Ginecol Obstet México, 70, 510-520. 